\title{
SOP 07a: Monitoring of a Trial under CESAR Responsibility
}

\section{Background}

Core 'SOP 07: Data Flow, Monitoring, and Archiving' was written under the assumption that a trial is sponsored by a pharmaceutical company that also takes the responsibility of monitoring according to Good Clinical Practice (GCP). The SOP 07a is a supplement to SOP 07 and describes the monitoring procedures in more detail. It is applicable if

- The supplier of an investigational drug decides to contract the monitoring responsibility to CESAR, or if

- CESAR decides to conduct a trial under its sole responsibility, including the sponsor responsibility of ensuring trial monitoring.

The Chair of a CESAR working group in close cooperation with the Coordinating Investigator (CI) of a trial has to ensure that appropriately instructed, trained, and experienced persons, e.g. a clinical research associate or a research nurse with particular experience in the conduct of clinical trials according to GCP, are available to perform the monitoring activities defined in this SOP.

\section{General Considerations and Procedures}

All monitoring activities, including phone calls, must be documented in writing. A report template may be used as guide or checklist for items to be covered during a site visit. Where appropriate and required, a template may be adapted to the requirements of a particular study.

Available report templates should be used for reporting of visits or other contacts. Relevant observations on items not covered by a generic report template should be reported as well, using an attachment, as applicable. Each report has to be submitted to a supervisor (e.g. the CI or his/her designee) for review.

Investigators and relevant site personnel should be notified, in writing, about problems noted that require corrective $\operatorname{action}(\mathrm{s})$.
For each clinical trial site a trial initiation visit will be performed. The visit should be as close to the start as possible, preferably after receipt of the trial medications at the site but before first drug administration to trial subjects. Each clinical trial should be monitored during its active phase. Each visit to an investigator's site will be recorded in a Monitoring Log form, indicating visitor, date, and purpose of the visit.

The first monitoring visit should be performed soon after the investigator has enrolled (randomized) trial subjects (patients) and the data are available for review. Subsequent monitoring visits should be conducted at a regular interval.

The frequency of monitoring visits may be adjusted depending on the nature of the trial, the enrollment rate, and the performance of the site.

The closeout visit should be performed as soon as possible after the last subject (patient) has completed the trial procedures at that trial site.

\section{Activities before a Trial Initiation Visit}

Activities before a trial initiation visit will include the following:

- Check which regulatory documents, if any, are outstanding.

- Ensure that the following items have been shipped to the investigational site or that shipping arrangements are made for

- Investigational product(s) ('trial medication'),

- Case Report Forms (CRFs),

- Investigator's file,

- Additional trial-specific materials, if needed (e.g. laboratory supplies, specimen containers, special forms, equipment).

- Contact the investigator and/or trial coordinator to schedule a mutually convenient time for the trial initia- 
tion visit. Every effort should be made to include in the visit pertinent site personnel, like investigator, study coordinator/nurse, pharmacist, and sub-investigator(s) as applicable.

- Review budget and proposed payment schedule (e.g. financial agreement or contract).

\section{Activities during a Trial Initiation Visit}

- Record the visit in the Monitoring Log.

- The trial initiation visit will include the review of the following:

- Clinical trial protocol and trial conduct,

- CRFs,

- Investigator files, including essential documents to be retained,

- Investigation product(s) and other trial-specific material(s) if applicable,

- Regulatory compliance (including identification of source documentation, adverse event (AE) reporting procedures and authorized study personnel),

- Emergency code break if applicable,

- Frequency of monitoring visits.

- As necessary, the following will be re-evaluated:

- Investigational site facilities,

- Facilities for storage of investigational product(s),

- Time frame for the trial in relation to subject selection criteria and availability,

- Any trial-related issues.

- Retrieve outstanding trial and regulatory documents.

\section{Activities Following a Trial Initiation Visit}

- Complete the Initiation Visit Report and forward it to the superior for review.

- Write a follow-up letter to investigator (or study personnel):

- Providing requested information,

- Requesting any additional information and action items as necessary,

- Summarize the relevant findings of the trial initiation visit within the letter or an attached copy of the Initiation Visit Report, for inclusion into the investigator's file.

- Arrange for initial study payment (if appropriate).

- File the Initiation Visit Report, regulatory and trial documents, and any correspondence.

\section{Activities before a Monitoring Visit}

- Review the following items to ensure that appropriate actions are taken and outstanding documents are retrieved during the monitoring visit:
- Previous monitoring visit reports or contacts for outstanding issues,

- Current enrolment status - reported serious adverse events (SAEs),

- Trial supplies provided so far,

- Data discrepancy forms (DDFs) or data query forms and working copies of CRFs, if available,

- Outstanding data issues,

- Outstanding laboratory issues,

- Outstanding regulatory documents, if any.

- Contact the investigator and/or trial coordinator to schedule a mutually convenient time for the trial monitoring visit and to identify items that should be sorted out before the monitoring visit. Request for the visit, as applicable:

- Participation of pertinent site personnel (i.e. investigator and/or sub-investigator, study coordinator, nurse, and pharmacist),

- Availability of applicable documents for review (i.e. signed Informed Consent forms, CRFs, etc.),

- Outstanding documents or issues to be resolved during the monitoring or to be completed or collected for review and/or retrieval.

- Gather any documents or materials necessary for the visit.

\section{Activities during a Monitoring Visit}

- Record the visit in the Monitoring Log.

- Review/assess the following:

- Enrolment status, rate of enrolment, and drop-outs,

- CRFs and data query forms if applicable,

- Source documents and informed consent forms; AEs and SAEs,

- Investigational products (i.e. amount on stock, retest/expiry date, storage conditions, dispensing logs),

- Protocol compliance (e.g. selection criteria, dosing schedule, concomitant treatments, laboratory tests, and sampling),

- Regulatory compliance (e.g. investigator's file up to date; continued acceptability of the site, personnel, and equipment; laboratory certificates and normal ranges up to date; SAE reporting up to date; Institutional Review Board (IRB) or Ethics Committee submissions and approval up to date),

- Retrieve outstanding trial and regulatory documents, if any, as well as completed and verified CRFs according to individual trial requirements.

- Discuss with the investigator and staff - if possible during the same visit - discrepancies and recommended corrective action(s). 


\section{Activities Following a Monitoring Visit}

- Complete the Monitoring Visit Report and forward it to the supervisor for review.

- Write a follow-up letter to investigator (or study personnel):

- Summarize the relevant findings of the monitoring visit,

- Provide requested information,

- Request any additional information as necessary.

- Arrange for interim payments, if appropriate.

- Forward retrieved CRFs for data processing.

- File the Monitoring Visit Report, retrieved regulatory and other trial documents, and any correspondence.

\section{Activities before a Closeout Visit}

- Review the following items to ensure that appropriate actions are taken and outstanding documents are retrieved during the closeout visit:

- Previous Monitoring Visit Reports or contacts for outstanding issues,

- Enrolment status,

- Reported SAEs,

- Investigational products provided,

- Data queries and working copies of CRFs, if available,

- Outstanding data issues,

- Outstanding laboratory issues,

- Outstanding regulatory documents, if any.

- Discuss with appropriate trial medication owner's personnel the requirements for shipment of investigational products and other materials, as applicable.

- Contact the investigator and/or trial coordinator to schedule a mutually convenient time for the trial closeout visit.
- Request remaining regulatory and other documents to be completed and made available for review/retrieval, including:

- Patient Identification List (for checking purpose only: compare with available CRFs),

- CRFs,

- Data discrepancy forms as far as available,

- Laboratory reports,

- Study medication records,

- Final information on SAEs.

\section{Activities during a Closeout Visit}

- Record the visit in the Monitoring Log.

- Review/assess the following:

- (To be defined specifically for each study).

- Retrieve outstanding trial and regulatory documents, if any, as well as completed and verified CRFs.

- Discuss with the investigator and the staff discrepancies and recommended corrective action(s).

\section{Activities Following a Closeout Visit}

- Complete the Closeout Visit Report and forward it to the supervisor for review.

- Write a follow-up letter to investigator(s)/study personnel outlining final disposition of the study to include information regarding retention of documents, expectation of audits or inspections by regulatory authorities and submission of a final report to the IRB and a copy provided to the $\mathrm{CI}$.

- Verify receipt of return drug shipment.

- Forward retrieved CRFs for data processing.

- Arrange for final payments related to the site.

- File the Closeout Visit Report, retrieved regulatory and other trial documents, and any correspondence. 